\title{
Rethinking Research Methods: Introduction to the Special Edition
}

\author{
By Robert Lawrence McKenzie and Mira Mohsini (School of Oriental and African Studies)
}

\section{Purpose and Scope}

This special edition of Anthropology Matters focuses on a fundamental question that virtually all social scientists encounter, namely how to conduct research on any given topic. Although this question appears straightforward at first pass, practical experience demonstrates the theoretical and pragmatic complexities involved in conducting research.

Over the past few decades, the manner in which social scientists have addressed the question of how to conduct research has shifted tremendously. Early ethnographic studies set out to examine and elaborate "objects" of inquiry, sometimes perceived as ahistoric and rigidly bounded fieldwork sites that could be comprehensively "known." As the social sciences began to transition away from positivist frameworks, research methodologies were compelled to adapt to the shifting boundaries between "the field," fieldwork, and the fieldworker.

Anthropology, in particular, encountered several theoretical turns, challenging previous ways of knowing and bringing to the fore questions of methodological import. With every so-called turn that a discipline endures, new boundaries are constructed and limitations are tested and established. The aim of this special edition is to explore and expand these boundaries by focusing on the theoretical and practical complexities of research methods.

All of the papers in this issue make important contributions to our understanding of research methods and broader methodological concerns. The papers examine specific methods employed during fieldwork as well as comment on their connection to methodological paradigms that are often largely shaped by disciplinary histories, practices, and deeply entrenched epistemologies. The impetus for bringing these diverse papers together emerged after we organised an international conference on the same topic. The conference, aptly entitled "Exploring and Expanding the Boundaries of Research Methods," took place over two days on 31 October and 1 November 2008.

The conference brought together nearly one hundred and fifty doctoral students and academics from some thirty-five institutions in North America, Europe, and Asia, and it was viewed by even more, as both conference days were live-streamed via the Internet. In addition to sixteen paper presentations, there were two film screenings - one on masonry builders in Djenne, Mali and the other on the lived experience of undocumented refugees in Europe. The high turn-out of attendees (including virtual attendees) and the ensuing lively discussions indicated the timeliness and importance of research methods and their ethical implications. At the conclusion of the conference there was a decidedly strong interest to explore and develop these themes in a collection of papers. 
The idea behind the conference (and by extension this special edition) was to assemble a diverse group of scholars from the social sciences and humanities in an effort to facilitate dialogue about research methods ranging from "traditional" techniques, such as interviewing and surveying, participant observation and focus groups, to more "experimental" practices incorporating film, photography and sound. Despite participants coming from different academic disciplines - anthropology, government, architecture, development studies, history, geography and sociology - the consensus was that, beyond formal training and discussions with colleagues, research methods are often learned, tried and tested on the job, that is, while in the process of doing research. There is little debate that such learning has its advantages, but there are also inherent disadvantages. In fact, a common theme during the conference was that the particularities of a context and the circumstances of a given research locale inevitably influence (and sometimes adversely so) the choice of methods employed and yet researchers are often unprepared for such contingencies. Indeed, many researchers at the conference discussed how they completely overhauled a well-structured and well-planned research agenda due to the unpredictability involved in conducting fieldwork.

The papers selected for this special edition reflect not only on the diversity of methods, but also on the ways in which methods and their ethical implications evolve during the course of conducting research. Therefore a key issue in this collection is that research methods are as much about the tools researchers employ as they are about the implications, disturbances and even failures that result in their use. While the papers address these thorny issues, there is no attempt to provide definitive solutions. Rather this collection should be viewed as an opportunity for additional dialogue and we hope that these papers will offer useful reference points for future doctoral students and researchers embarking on new projects.

A conference of this magnitude could not have been possible without considerable support and we would be remiss not to identify some of the institutions and individuals involved. We are grateful for the generous financial assistance from the Royal Anthropological Institute, the Arts and Humanities Research Council, the Department of Anthropology and Sociology at the School of Oriental and African Studies (SOAS), the SOAS Faculty of Arts and Humanities, and the Brunei Gallery in London, where the conference was hosted. We would like to thank Professor Richard Fardon, Head of the Department of Anthropology and Sociology at SOAS, who supported this conference from the moment we suggested it to him, nearly one year before it took place. We would also like to thank Dr. Andrew Irving, RCUK Research Fellow at the University of Manchester, and Dr. Desmond Thomas, Research Skills Coordinator in the Academic Development Directorate at SOAS, for their creative and supportive ideas as we organised and prepared for the conference. We must also thank Dr. Irving and Dr. John Campbell, Senior Lecturer in the Department of Anthropology and Sociology at SOAS, for their active participation in the conference and for their thoughtprovoking papers in this special edition. Lastly we would like to thank Mr. Jens Franz for his patience and technical support, without which the conference could not have been livestreamed globally nor would it have been possible to create a Website on its key themes (see http://expandingboundaries.wordpress.com/).

\section{Outline and Overview of Papers}

For this special edition we are privileged to present papers from doctoral students and three established academics (see contributions from Webley, Campbell and Irving). Most of the papers were presented during the conference, while some were solicited and have been 
specially written for this edition of Anthropology Matters (see contributions from Campbell and Irving).

The Foreword by Professor Paul Webley, Director and Principal of SOAS, is adapted from his insightful opening remarks at the conference. His paper wonderfully illustrates the creative and innovative capacity of research methods to bring different disciplinary norms into dialogue. Using examples from his own field of economic psychology, Webley discusses the benefits and novel insights that can emerge from exploring a range of research methods even within the parameters of a single research project. Webley suggests that this may take us beyond our comfort zone, but in the process we create boundary-pushing collaborations that make research all the more relevant, a point that is expressly or implicitly covered in this edition.

Following Prof. Webley's paper, the edition is divided into two parts. The first part includes papers that contribute theoretically to debates on research methods and their ethical implications. The second part includes papers that predominantly focus on methodological concerns. The special edition concludes with a paper by Dr. Andrew Irving. While we decided to organise the papers according to this structure, one need not read them in successive order. Each contribution offers important theoretical and methodological insights into the underlying question that informed this project, namely how do we, as researchers, come to know - through the application of research methodology - our chosen topic of study?

\section{Theoretical Concerns}

In the first paper, Hayder Al-Mohammad challenges researchers to (re)consider epistemological and methodological boundaries. Al-Mohammad is concerned with the implications of theoretical oversights resulting from reliance on apprenticeship and/or mimesis. Reliance on such methods, Al-Mohammed contends, leads to "categorical errors and overly hasty commitments to metaphysical conclusions." Along these lines, AlMohammed raises the simple but profoundly important question: "In putting myself, my body, through the same apprenticeship as another do I get insight into the process and/or the experience of those who have undertaken the apprenticeship?" Regardless of how one answers this question, one cannot deny the challenge that Al-Mohammed puts to researchers, namely that we must (re)consider the boundaries of our abilities to know and understand the experiences of Others. By not questioning these boundaries researchers run the risk of conflating epistemic and categorical questions with methodological problems.

The second contribution by Dr. John Campbell is a provocative and timely paper that explores questions of ethics and their potential implications for anthropology. While several historic moments have triggered ethical concerns, Campbell contends that such concerns have rarely received their due attention and debate. Campbell explores these thorny issues from three perspectives. Firstly, through the prism of the academy, he traces the development of a formal code of ethics by the American Anthropological Association, a protocol that emerged comparatively late and in reaction to outside attacks on the discipline. Secondly, Campbell explores the ethical questions raised by the changing dynamics between researcher and informant due to the development of "studying up" within powerful institutions - a shift that has enabled informants to challenge the interpretations of fieldwork outcomes. Thirdly, Campbell demonstrates that ethics extend far beyond the rigid boundaries of codes of 
conduct, precisely because these codes may not provide sufficient guidelines to protect both researcher and his/her informants against "various agents of the state who now take an interest in what we have to say and how and when we talk about our research." Campbell's primary contention is that we - individually and collectively - must consider the myriad ethical concerns and take a "hard look at our profession and at our own practices."

\section{Methodological Concerns}

The second part of the edition includes five papers that focus on a variety of research methods employed in vastly different contexts. Suzanne Hall's paper focuses on café culture along one road in South London. Trained as an architect, Hall combines methods from her discipline with ethnographic methods to understand manifestations of difference and sociability within the spatial make-up of a diverse, multi-ethnic locale. Within the dense spatial configurations of an urban street, Hall uses techniques of juxtaposition, layering, and collage in order to research, write, and understand (or frame) difference. Through the use of innovative methods of seeing and writing, Hall's insightful contribution addresses the challenges faced by many researchers about how to represent the complexity of difference and how to capture the socio-spatial relationships between individuals, groups and place.

Jennifer O'Brien's paper skilfully illustrates the increasing institutionalisation of certain "traditional" research methods and the need to employ creative methods, particularly when researching sensitive topics. O'Brien explores how to meaningfully discuss the spread of HIV/AIDS with young people in Uganda. She demonstrates the failure of institutionalised methods, such as questionnaires and focus groups, to gain access to and information from these groups. O'Brien discusses how the introduction of a game, and the use of her positionality as a resident of a village, afforded her unique access to young people. The paper seeks to expand the definition of what constitutes research methods beyond their institutional manifestations, which have become a significant aspect of researchers' repertoires. A key lesson learned is that identifying alternative methodological tools can bridge socio-cultural differences and create spaces that encourage and support the development of rapport between researcher and informants.

In the next paper, Ruth Goldstein examines different ways of knowing the field. Through ethnographic fieldwork in Mali, Goldstein explores how dancing, drumming, language and storytelling transmit deeply held beliefs about history, culture and current events. By exploring how international NGOs sometimes (mis)communicate messages about sexual health knowledge, Goldstein encourages us to recognise and respect differences in seeing, being, and listening. She demonstrates that these differences present methodological imperatives and have far-reaching implications for both social science research and international aid programmes.

Sarah Osterhoudt's paper examines the creative possibilities of moving beyond disciplinary boundaries by employing insights from the field of literature and applying these to anthropological debates. Osterhoudt explores the short stories of Jorge Luis Borges and their relevance to important practical and ethical questions in anthropology. She suggests that the often imaginative and magical realist stories of Borges can provide powerful allegories to some of anthropology's most prevalent, but also contested practices, including ethnographic research, documentation and representation. Osterhoudt's paper reminds us that bounded and institutionalised disciplines cannot claim proprietary rights to ideas, knowledge, and debates. 
In fact, crossing these boundaries serves to deepen and expand our understanding of our own disciplinary research methods.

In the last paper of this section, Johanna Söderström examines the advantages and disadvantages of using focus groups as a key method to help understand the meaning of elections among ex-combatants in the post-conflict environment of Liberia. Söderström's paper illustrates the unpredictable yet insightful tensions that can arise during focus group sessions. These sessions allow for constructive interactions and novel forms of information to be generated in controlled research settings. Söderström argues that building trust among focus group participants is an essential factor for both cultivating relationships and ensuring successful research outcomes. Like other papers in this collection Söderström explores how trust is a crucial, but often elusive and contingent factor in research.

\section{Conclusions}

To conclude this special edition, Dr. Andrew Irving has written a fascinating paper that challenges the notion of boundaries as limits. He explores how boundaries can shape and influence the conditions for creativity, thus leading to the possibility of transgressing their very instantiation. The realisation of such boundary-crossing possibilities, Irving suggests, means that, "habits, conventions, institutions and human nature are not fixed and unchanging but open to transformation and intervention." The fluidity of boundaries, as opposed to the rigidity of limits, also applies to disciplines that seek to use and develop methodologies for representing the contemporary world. Irving argues that by looking at alternate histories of the emergence and trajectories of academic disciplines - in this case, anthropology - we can and perhaps should challenge assumptions about rigid methodologies and practices.

As a coda to this introduction, we would like to return to Professor Paul Webley's concluding remarks in his Foreword. If we are to explore and expand research boundaries, Prof. Webley invites us to reflect on our own practices as researchers and to move beyond our comfort zones by embracing other disciplinary research methods. In doing so, we will be better prepared to foster debates that push disciplinary practices into dialogue and engage with theoretical, practical and ethical issues centred around the complex world in which we live.

\section{About the Authors}

Robert Lawrence McKenzie is a $\mathrm{PhD}$ candidate at the School of Oriental and African Studies. His thesis, entitled "Against Refugees," is an ethnography of asylum-seeking amongst subSaharan men in Cairo, Egypt, one of the world's largest urban environments for refugees. His thesis explores the historical events and political processes - including the roles of domestic and international actors - that have shaped and institutionalised the modern asylumsystem in Egypt. His research draws special attention to the macro-power forces that influence human action. Robert can be contacted at bmck15(AT)gmail.com.

Mira Mohsini completed a PhD in Anthropology at the School of Oriental and African Studies in 2010 entitled "Becoming an 'Asli Karigar': The Production of Authenticity among Old Delhi's Muslim Artisans." Her research interests include marginalisation, agency, Islam in South Asia, embodiment, globalisation and consumption. Mira can be contacted at mjmohsini(AT)gmail.com. 